\title{
Classification of ATP binding sites using sequence-based fingerprints and molecular interaction fields J Dreher* and K Baumann
}

Address: Institut für Pharmazeutische Chemie, Technische Universität Braunschweig, Beethovenstr. 55, 38106 Braunschweig, Germany

* Corresponding author

from 4th German Conference on Chemoinformatics

Goslar, Germany. 9-II November 2008

Published: 5 June 2009

Chemistry Central Journal 2009, 3(Suppl I):P77 doi:I0.II86/I752-I53X-3-SI-P77

This abstract is available from: http://www.journal.chemistrycentral.com/content/3/SI/P77

(c) 2009 Dreher and Baumann; licensee BioMed Central Ltd.

Protein kinases represent one of the largest groups of drug targets in humans. All kinase enzymes share a catalytic domain that binds ATP. In recent years, many small molecule ATP-competitive kinase inhibitors have been developed. However, the evolutionary relatedness and structural conservation of these targets often lead to unforeseen cross reactivity.

The goal of this work was to develop ATP binding site descriptors that reflect the pharmacological profile of a predefined set of inhibitor molecules. For this purpose, two different ways of representing the binding site were compared: Sequence based fingerprints and descriptors based on Molecular Interaction Fields (MIFs). To achieve comparability of the different binding sites, the binding pockets needed to be superimposed. Again, two different methods were compared: Structure based techniques and pharmacophore based techniques.

A dataset of 38 kinase inhibitors with experimental data against 90 human kinases was used to assess the performance of the developed descriptors [1]. Performance was measured by several neighbourhood behaviour criteria [2] which are based on a comparison of the pairwise distances of the binding site descriptors to the pairwise distances of the inhibitor's pharmacological profiles.

Overall, classification based on the sequence of the binding pocket performed best. However, sequence-based methods cannot detect unrelated kinases with similar pharmacological profile. This in turn is the strength of the MIF-based descriptors since these descriptors characterize the geometry of hot spots on the protein and are independent of sequence information. In addition to classification tasks, MIF-based descriptors can be used to derive structure-based pharmacophores that highlight the different selectivity profiles of the binding pockets.

\section{References}

I. Karaman MW, Herrgard S, Treiber DK, et al.: A quantitative analysis of kinase inhibitor selectivity. Nature Biotechnology 2008, 26: $127-132$.

2. Horvarth $D$, Jeandenans $C$ : Neighborhood behavior of in silico structural spaces with respect to in vitro activity spaces-a novel understanding of the molecular similarity principle in the context of multiple receptor binding profiles. J Chem Inf Comput Sci 2003, 43(2):680-690. 